\title{
Gender effect on underwater undulatory swimming technique of young competitive swimmers
}

\author{
ŁUKASZ WĄDRZYK ${ }^{1 *}$, ROBERT STASZKIEWICZ ${ }^{1}$, ŁUKASZ KRYST $^{2}$, MAGDALENA ŻEGLEŃ $^{2}$ \\ ${ }^{1}$ Department of Biomechanics, Institute of Biomedical Sciences, University of Physical Education in Kraków, Poland. \\ ${ }^{2}$ Department of Anthropology, Institute of Biomedical Sciences, University of Physical Education in Kraków, Poland.
}

\begin{abstract}
Purpose: The study aim was determining gender-related differences of underwater undulatory swimming (UUS) kinematic indicators and their impact on UUS velocity. Methods: The study included 18 girls (F: age $16.71 \pm 0.64$ years, FINA points $551 \pm 68$ ) and 23 boys (M: age $16.79 \pm 0.57$ years, FINA points $533 \pm 66$ ) training swimming. After marking characteristic anatomical points, subjects performed approximately 7 meters of UUS. A filming device placed behind the underwater window registered the trial. Recordings were analysed using the SkillSpector programme. Results: Boys swam faster (F: $1.24 \mathrm{~m} / \mathrm{s}, \mathrm{M}: 1.35 \mathrm{~m} / \mathrm{s})$, overcame a greater distance during one cycle (F: $0.67 \mathrm{~m}, \mathrm{M}: 0.74 \mathrm{~m})$, performed movements with higher toes amplitude (F: $0.58 \mathrm{~m}, \mathrm{M}: 0.63 \mathrm{~m})$, obtained higher amplitude and frequency product (F: 1.05, M: 1.15) and smaller ankle joint range of motion (F: $\left.64^{\circ}, \mathrm{M}^{\circ} 57^{\circ}\right)$. In both groups, relationships between velocity and: maximal ankle joint extension, distance covered during one cycle and backward toes shift during downward movement were found. The results were statistically significant $(p<0.05)$. Conclusions: Girls and boys differed in kinematic indicator level, but UUS velocity depends on identical kinematic variables, meaning UUS technical training can be performed without gender-division.
\end{abstract}

Key words: biomechanics, swimming, gender differences, underwater undulatory swimming

\section{Introduction}

Swimming is one of the most dynamically developing sports disciplines, as evidenced by regular improvement of world records in all competitions at the most important swimming events [14]. There are many reasons for the constant progression of results. These include systemic solutions, such as the popularisation of sport among children and youth, conducive to the proper selection for the sport of swimming [24]. Technological progress in areas used in swimming competitions is not without significance [14]. Within this respect, changes in swimming rules are also beneficial, enabling, among others, overcoming distances in a less restrictive manner. Most of all, the training methods and control of their effects are improved [16]. Thanks to them, sports level among contemporary athletes is higher than in the past [14].
Planning the training process is based on the results of scientific research. Implementation of research results in the field of biomechanics, physiology or psychology favours the achievement of better results and provides the basis for rejecting many common opinions about swimming training [16]. Only scientific research allows to properly direct the training process and determine its priorities [16].

Researchers invariably use video methods for registration and subsequent kinematic analysis of a swimmer's movement [13]. Although this method is one of the oldest regarding studies in the field of biomechanics of swimming, preparation of the video material is still time-consuming. However, researchers do not resign from this method because it enables them to achieve extremely reliable results [5]. The reason for undertaking such research is the possibility for observing the course of the movement without interfering with it [13]. Currently, thanks to the

\footnotetext{
* Corresponding author: Łukasz Wądrzyk, University of Physical Education, al. Jana Pawła II 78, 31-571 Kraków, Poland. E-mail: wadrzyk504@gmail.com

Received: July 18th, 2019

Accepted for publication: September 16th, 2019
} 
popularisation of the most modern waterproof sports cameras, the area of scientific penetration in the field of swimming technique assessment has grown significantly [5].

Since the beginning of the 21st century, a number of studies have been carried out on underwater undulatory swimming [4], [9]. As Atkison et al. [4] point out, this element of the swimming race significantly influences the final result achieved by competitors. This is due to the fact that swimmers move this way after the start and after every turn in almost all races. The exception is the breaststroke, for which the underwater phase is limited by swimming regulations [10].

The coach's evaluation of underwater undulatory swimming is limited, because in everyday training practice, the coaches rarely use underwater motion observation. For this reason, many studies have been undertaken to characterise the technique in question. Among others, the description of underwater undulatory swimming among athletes at different ages and various levels of training has been performed [3]. Many researchers, including Arellano et al. [2] and Atkison et al. [4] divided the cycle of underwater undulatory swimming into phases. Typically, the distinction of such division was vertical displacement of the toes [9]. In this way, the upward and downward movements were distinguished, while describing both phases using kinematic variables [4], [9], [10]. On this basis, the similarities and differences of the collected spatial-temporal indicators were assessed. In light of this, the course of the underwater undulatory swimming carried out by humans and some water mammals was also compared [25]. In the course of these studies, it was found that achieving high swimming velocity with the use of underwater undulatory swimming is possible, among others, due to the increase in the vertical velocity of the toes (increase in the frequency of movements while maintaining their appropriate breadth), large plantar and small knee joint flexion as well as maintaining proper spatial-temporal relationships between individual segments [7], [10], [22]. Also important was the observation that the values of kinematic variables describing the upward and downward movements were definitely similar to each other [4], [9].

Regular practice in research on underwater undulatory swimming is the inclusion of both sexes in the study groups. It is the reason why there is no description of differences in the technique of their performance by men and women. Only Arellano et al. [2] and von Loebbecke et al. [25] made such distinction, but they did not show differences in the kinematic indica- tors recorded in both sexes. It should be noted, however, that these studies were conducted among a relatively small number of subjects for whom a limited number of kinematic variables were analysed.

Differences in somatic structure and the level of motor abilities give rise to assumptions about the differences in the technique of underwater undulatory swimming in both sexes [6]. They may be indications that the patterns of underwater movements to which male and female swimmers should strive are also different. So far, however, it has not been established whether the effectiveness of underwater undulatory swimming by athletes of both sexes is characterised by the same type of kinematic indicators.

The aim of this study was to characterise differences in the technique of underwater undulatory swimming depending on sex. In addition, an attempt was made to describe factors affecting the effectiveness of underwater undulatory swimming. The following hypotheses were verified:

1. Male swimmers swim faster with the use of underwater undulatory swimming.

2. Swimmers of both sexes differ in the level of kinematic indicators describing underwater undulatory swimming.

3. For both sexes, the effectiveness of underwater undulatory swimming can be characterised by the same kinematic variables.

4. Technical training of underwater undulatory swimming can be carried out without division into gender.

\section{Materials and methods}

The research was carried out at the Indoor Swimming Pool Complex of the University of Physical Education in Kraków. Measurements were performed on an 8-lane pool with a length of $25 \mathrm{~m}$, equipped with a window enabling observation of swimmers' underwater movements.

The group of subjects consisted of 23 boys and 18 girls aged 15-18. All participants of the study trained on a daily basis at the School of Sports Championships in Oświęcim. The average weekly training volume of the swimmers was $18 \mathrm{~h}$ in water and $6 \mathrm{~h}$ on land. On the day of testing, all competitors had the appropriate medical examination qualificating them to participate in professional swimming training. In addition, each participant and/or their legal guardian, after being familiarised with the measurement procedure, expressed their written consent to participate in research in the formula described below. To carry out 
the measurements, the consent of the Bioethical Commission at the District Medical Chamber (authorisation number: 3/KBL/OIL/2018) was obtained.

The basic characteristics of the examined boys and girls are presented in Table 1. The sport level of the subjects was determined on the basis of the best result achieved by the competitor for the distance of $100 \mathrm{~m}$ freestyle calculated into FINA points. These data were downloaded from the website of the Worldwide Swimming Ranking. The significance of differences between the mean values of these variables (tests for independent samples) was also assessed.

Table 1. Characteristics of the examined group ( $\mathrm{F}$ - girls, $\mathrm{M}$ - boys)

\begin{tabular}{|l|c|c|c|}
\hline & $\mathrm{F}$ & $\mathrm{M}$ & $p$-value \\
\hline Age [years] & $16.71 \pm 0.64$ & $16.79 \pm 0.57$ & 0.71 \\
\hline Body height [m] & $1.67 \pm 0.06$ & $1.81 \pm 0.06$ & $\mathbf{0 . 0 0}$ \\
\hline Body mass [kg] & $61.57 \pm 8.81$ & $72.78 \pm 9.37$ & $\mathbf{0 . 0 0 *}$ \\
\hline Sports level [FINA points] & $551 \pm 68$ & $533 \pm 66$ & 0.40 \\
\hline
\end{tabular}

The research method was similar to that described in earlier study [21]. Before the measurements were performed, the centers of rotation of the: upper, knee, hip, shoulder, elbow and radial-wrist joints were marked with a waterproof marker on the left, lateral side of the body. The V toe of the left foot and the V finger of left hand were also marked on the lateral side. These markings were performed in such a way as to be visible from a minimum distance of $10 \mathrm{~m}$.

After a short warm-up on land and in water, the subjects were familiarised with the measurement procedure. The task of the participants was to swim the section of approximately $7 \mathrm{~m}$ three times at a depth of about $1 \mathrm{~m}$ below the surface of the water with the use of underwater undulatory swimming. The subjects were instructed to overcome the distance at maximal speed. Between each trial, all subjects had approximately 3 minutes of rest.

All trials were recorded using the Casio Exilim EX-FH25 recording device in the filming mode at a frequency of 120 frames/s. The device was placed on a tripod behind the underwater window at a depth of about $1 \mathrm{~m}$ below water level, at a distance of about $8 \mathrm{~m}$ from the tested participant. The camera lens was set perpendicularly to the direction of movement of the subjects. The described arrangement of the measurement line was consistent with previous research by the authors of this study [21].

The described setting enabled underwater recording of an approx. 5-meter length area, thus, allowing to film a minimum of three full underwater undulatory swimming cycles. For the purpose of this study, it was assumed that the cycle began when the $\mathrm{V}$ toe of the foot was at its lowest position, while the end of the cycle occurred when the $\mathrm{V}$ toe ended the downward movement.

After recording all of the trials, in the middle of the measurement area, parallel to the water surface, a calibration frame was placed, the recording of which was later used to scale the recordings.

The video material was analysed using the SkillSpector computer programme. A 10-point model dividing the body into 8 segments was used for kinematic analysis. First of all, for each trial, the average horizontal velocity at which the subjects moved during underwater undulatory swimming was determined. Only the recording on which the subject moved the fastest was considered for further analysis. Based on it, the following variables were determined:

- average horizontal velocity of center of mass $[\mathrm{m} / \mathrm{s}]$ $-v_{\mathrm{COM}}$,

- maximal flexion of the ankle joints $\left[{ }^{\circ}\right]-A_{\mathrm{FLEX}}$,

- maximal extension of the ankle joints $\left[^{\circ}\right]-A_{\mathrm{EXT}}$,

- range of motion of the ankle joints $\left[{ }^{\circ}\right]-A_{\mathrm{ROM}}$,

- frequency of movements $[\mathrm{Hz}]-f$,

- amplitude of toes movements - the difference between their highest and lowest position in one cycle $[\mathrm{m}]-A$,

- distance per cycle - horizontal distance covered by the center of mass during one complete cycle [m] - DPC,

- range of motion of the knee joints $\left[{ }^{\circ}\right]-K_{\mathrm{ROM}}$,

- horizontal displacement of toes during downbeat - backward foot displacement during downward movement of the limbs [m] - Toe DISP,

- product of amplitude and frequency $[\mathrm{n}]-I_{\mathrm{Af}}$.

Statistical analysis of the collected data was performed using the Statistica programme. For each variable, the mean was calculated and the measures of dispersion were determined. Subsequently, the normality of the distribution was analysed with the Kolmogorov-Smirnov and Lilliefors tests. Assessment of differences between boys and girls was conducted using the Student's $t$-test (in the case of close-tonormal distribution) or the Mann-Whitney $U$-test (when the distribution deviated from the normal level). For all of the above-mentioned operations, $p<$ 0.05 was assumed as the level of statistical significance. Furthermore, Cohen's $d$ effect size calculations were performed.

In order to determine the relationship between $v_{\mathrm{COM}}$ and the remaining kinematic variables, calculations of Pearson's $r$ linear correlation coefficient were performed, assuming the significance level of $p<0.05$. 
The final step in statistical analysis was to compare the correlation coefficients in the groups of girls and boys by means of the one-tailed test of differences.

\section{Results}

The values of kinematic variables describing the underwater undulatory swimming of girls and boys are presented in Table 2 . The mean horizontal velocity of the center of mass $\left(v_{\mathrm{COM}}\right)$ in boys was $1.35 \mathrm{~m} / \mathrm{s}$, and it was by $0.11 \mathrm{~m} / \mathrm{s}$ higher than in girls $(p<0.05)$. Other kinematic movement indicators also differed, but only some of these differences were significant. All variables describing angular changes in the ankle and knee joints were higher in girls, but only one of them $\left(A_{\mathrm{ROM}}\right)$ was significantly higher than that recorded in the group of boys. With the exception of horizontal displacement of toes during downbeat (Toe DISP), mean values of other variables were higher in the boys' group. Differences ranged from approx. $0 \%$ (range of motion of the knee joints) to approx. 20\% (maximal extension of the ankle joints).
In Table 3, the results of correlation analysis regarding the relationship between horizontal velocity $\left(v_{\mathrm{COM}}\right)$ and other kinematic variables characterising underwater movements of swimmers are presented. In the case of boys, the following were positively correlated with $v_{\text {COM }}$ values: maximal extension of the ankle joints $\left(A_{\mathrm{EXT}}\right)$, distance per cycle (DPC) and product of amplitude and frequency $\left(I_{\mathrm{Af}}\right)$. The correlation coefficient $r$ for the mentioned relationships ranged from approx. 0.48 to 0.61 . At the same time, $v_{\mathrm{COM}}$ and $\mathrm{Toe}_{\mathrm{DISP}}$ (horizontal displacement of toes during downbeat) formed significant, negative correlations $(r=-0.61)$. In the male group, no correlations were noted between $v_{\mathrm{COM}}$ and the range of motion in the ankle joints $\left(A_{\text {ROM }}\right)$, knee joints $\left(K_{\text {ROM }}\right)$, frequency $(f)$ and amplitude of movements $(A)$.

In the group of girls, the values of maximal flexion of the ankle joints $\left(A_{\mathrm{FLEX}}\right)$, range of motion of knee joints $\left(K_{\mathrm{ROM}}\right)$ and horizontal displacement of toes during downbeat (Toe ${ }_{\text {DISP }}$ ) were negatively correlated with the horizontal velocity of the center of mass. The last of the discussed indicators showed a particularly strong correlation with the $v_{\mathrm{COM}}$ value $(r=-0.817)$. A negative $r$ coefficient was also noted for the ampli-

Table 2. Values of kinematic variables describing underwater undulatory swimming in girls (F) and boys (M), taking differences between groups into account

\begin{tabular}{|l|c|c|c|c|c|}
\hline \multicolumn{1}{|c|}{ Variable } & $\begin{array}{c}\mathrm{F} \\
(\mathrm{mean} \pm \mathrm{SD})\end{array}$ & $\begin{array}{c}\mathrm{M} \\
(\text { mean } \pm \mathrm{SD})\end{array}$ & Relative difference & $p$-value & Effect size \\
\hline$v_{\mathrm{COM}}[\mathrm{m} / \mathrm{s}]$ & $1.24 \pm 0.12$ & $1.35 \pm 0.15$ & $9 \%$ & 0.01 & -0.80 \\
\hline$A_{\mathrm{FLEX}}\left[{ }^{\circ}\right]$ & $47.33 \pm 5.62$ & $44.32 \pm 5.10$ & $6 \%$ & 0.34 & 0.54 \\
\hline$A_{\mathrm{EXT}}\left[^{\circ}\right]$ & $16.60 \pm 8.58$ & $13.32 \pm 7.34$ & $20 \%$ & 0.19 & 0.40 \\
\hline$A_{\mathrm{ROM}}\left[^{\circ}\right]$ & $63.93 \pm 7.86$ & $57.64 \pm 8.50$ & $10 \%$ & 0.01 & 0.75 \\
\hline$f[\mathrm{~Hz}]$ & $1.83 \pm 0.20$ & $1.85 \pm 0.26$ & $1 \%$ & 0.84 & -0.09 \\
\hline$A[\mathrm{~m}]$ & $0.58 \pm 0.06$ & $0.63 \pm 0.07$ & $8 \%$ & 0.02 & -0.76 \\
\hline $\mathrm{DPC}[\mathrm{m}]$ & $0.67 \pm 0.10$ & $0.74 \pm 0.11$ & $10 \%$ & 0.03 & -0.65 \\
\hline$K_{\mathrm{ROM}}\left[{ }^{\circ}\right]$ & $76.20 \pm 9.34$ & $75.88 \pm 7.05$ & $0 \%$ & 0.90 & 0.04 \\
\hline $\mathrm{Toe}_{\mathrm{DISP}}[\mathrm{m}]$ & $0.14 \pm 0.05$ & $0.12 \pm 0.04$ & $14 \%$ & 0.17 & 0.43 \\
\hline$I_{\mathrm{Af}}[\mathrm{n}]$ & $1.05 \pm 0.09$ & $1.15 \pm 0.12$ & $9 \%$ & 0.002 & -0.94 \\
\hline
\end{tabular}

Although both groups of subjects were characterised by similar values of frequency of movements (about $1.8 \mathrm{~Hz}$ ), there was significant disproportion in their amplitude $(A)$. In this case, the boys reached an average amplitude approx. $0.05 \mathrm{~m}$ higher. As a consequence, the product of the amplitude and frequency of movements $\left(I_{\mathrm{Af}}\right)$ in boys also clearly showed higher values than in girls $(p<0.05)$. As already mentioned, male swimmers covered significantly longer distances (DPC higher by approx. $0.07 \mathrm{~m}$ ) during one cycle of underwater undulatory swimming. tude of movements $(A)$, but, in this case, the relationship was not significant. Correlations between horizontal velocity of underwater undulatory swimming and other variables were positive, however, only two of them (maximal extension of the ankle joints $-A_{\text {EXT }}$ and distance per cycle - DPC) were statistically significant. Similarly to the group of boys, no significant correlations were found between $v_{\mathrm{COM}}$, frequency $(f)$ and amplitude of toes movement $(A)$. The data from Table 3 also indicate that the directions of dependence between $v_{\mathrm{COM}}$ and other variables were similar for both sexes. The only exception was the amplitude 
of toes movement $(A)$, the values of which tended to create positive correlations with $v_{\mathrm{COM}}$ in boys, while in girls, they were negative.

Table 3. The value of correlation coefficients between $v_{\mathrm{COM}}$ and other variables, taking gender-related differences in their level into account ( $\mathrm{F}$ - girls, $\mathrm{M}$ - boys)

\begin{tabular}{|l|c|c|c|}
\hline Variable & $\mathrm{F}$ & $\mathrm{M}$ & $\begin{array}{c}p \text { value differences } \\
\text { in correlation coefficients }\end{array}$ \\
\hline$v_{\mathrm{COM}}$ & - & - & - \\
\hline$A_{\mathrm{FLEX}}$ & $-0.501^{*}$ & -0.206 & 0.162 \\
\hline$A_{\mathrm{EXT}}$ & $0.492^{*}$ & $0.510^{*}$ & 0.472 \\
\hline$A_{\mathrm{ROM}}$ & 0.178 & 0.316 & 0.334 \\
\hline$f$ & 0.459 & 0.204 & 0.201 \\
\hline$A$ & -0.137 & 0.291 & 0.104 \\
\hline $\mathrm{DPC}$ & $0.626^{* *}$ & $0.477^{*}$ & 0.266 \\
\hline$K_{\mathrm{ROM}}$ & $-0.714^{* *}$ & -0.281 & $0.042^{*}$ \\
\hline Toe & $-0.817^{* *}$ & $-0.610^{* *}$ & 0.103 \\
\hline$I_{\mathrm{Af}}$ & 0.411 & $0.612^{* *}$ & 0.213 \\
\hline
\end{tabular}

$* p<0.05$.

$* * p<0.01$.

In Table 3, the results of the test regarding differences between groups in correlation coefficients are also presented. It turned out that the range of motion of the knee joints $\left(K_{\mathrm{ROM}}\right)$ was the only indicator for which the correlation coefficients calculated for groups of girls and boys significantly differed $(p<0.05)$.

\section{Discussion}

The results of this study indicate significant differences in the technique of underwater undulatory swimming in both sexes. This can be stated, among others, on the basis of the greater swimming velocity with which the studied boys moved. In the literature, there are a number of reports on better results achieved by male athletes above the age of 14 [6]. Kenney et al. [11] estimated that in swimming, men obtain results better than women by an average of $7-11 \%$. The results of this study indicate that this may be the result of not only the described differences in full stroke swimming velocity [17], but also the efficiency of movement while underwater undulatory swimming. One of the reasons for the differences in sports results may be different hormonal reactions during puberty, as described by Wolański [23]. As a result, sexual dimorphism is strongly observed in bone growth, muscle and fat tissue [23]. The results of this study are fully consistent with the information that has appeared in previous publications.
Differences were noted not only in velocity, but also in other kinematic indicators describing the technique of underwater undulatory swimming, which is why the results require broader discussion. The analysis of gender-related differences is, however, restricted by the fact that until now the authors of the research devoted little attention to the characterisation of underwater undulatory swimming from in a dimorphic approach. Only Arellano et al. [2] and von Loebbecke et al. [25] took up this subject in their work, not finding differences in the course of movement among women and men. In both of the above-mentioned works, the study groups were relatively small, therefore, statistical analysis of the results did not allow for valuable inference. For this reason, in our research, the measurements included larger groups, and the results were also referred to the full stroke swimming techniques.

In addition to velocity, the most commonly used kinematic indicators serving the description of the full stroke movement of a swimmer is stroke length (SL distance covered during one cycle of the upper limbs) and stroke rate (SR - number of full cycles performed per minute) [26]. The product SL and SR enable to determine velocity of a swimmer. This means that they can achieve a higher velocity by simultaneously increasing the value of each of these variables or only one of them without modifying the level of the other one. It is worth mentioning, however, that increasing the length of stroke length is usually associated with a decrease in stroke rate [26].

Based on stroke length and pace, it is possible to evaluate the technique of full stroke swimming [26]. In this manner, men and women perform movements at a similar rate, as reported by Seifert et al. [17]. The authors mentioned also demonstrated that there are clear differences regarding stroke length in favour of men, which translates into higher swimming velocity achieved by them.

In the case of underwater undulatory swimming, SL and SR counterparts may be: distance per cycle (DPC) and frequency of movements $(f)$ [2], [4]. In our research, during one cycle, the boys overcame a distance more than by $10 \%$ longer than the girls ( $0.74 \mathrm{~m}$ and $0.67 \mathrm{~m}$, respectively). At the same time, in both study groups, there was a similar frequency of movements $(1.83 \mathrm{~Hz}$ in girls and $1.85 \mathrm{~Hz}$ in boys). These observations were, therefore, similar to those made for full strokes swimming [17]. It turned out that both during the movement of standard swimming techniques and underwater undulatory swimming, differences regarding gender in technique are highlighted in a similar way - through different distances covered per one cycle. 
The nature of the task performed by swimmers in this study (short duration, maximal intensity) gives grounds for classifying it as anaerobic exercise [11]. Many authors have documented significant differences between the results achieved by men and women in movement tasks with such a metabolic basis [6]. It seems that male athletes achieve better results mainly due to a higher proportion of lean body mass and a lower level of body fat [11], [23]. Morphological diversity of body build is also manifested in efforts of longer duration [11]. Somatic discrepancies have an impact on gender-related differences in the range of sports results in swimming short and long distances, which has been documented, among others, by Kenney et al. [11]. The results of our research also indicate that in the technique of underwater undulatory swimming, the discussed aspects have significant impact not only on the swimmers' velocity, but on other kinematic indicators as well.

According to Kjendlie and Stallman [12] and Kenney et al. [11], larger body dimensions may have a positive effect on sports success, among others, in swimming. In the case of full stroke swimming, the impact of somatic build on results has been documented, among others, by Kjendlie and Stallman [12]. It has been proven that taller swimmers, with longer upper limbs and larger hand surfaces, are able to swim standard techniques faster than shorter swimmers. Nevill et al. [15] also point out that even the ratio of the length of the main propelling body parts (hands, feet) to the length of other segments of the upper and lower limbs may have impact on swimming velocity in the case of full stroke swimming. Thus, the effectiveness of swimmers may be dependent on the length of the thigh and shank as well as the surface of the feet. Perhaps this is why in the presented work, the male swimmers, being taller than girls by approximately $0.14 \mathrm{~m}$, were able to swim faster. However, the assessment of the impact of somatic build on the effectiveness of underwater undulatory swimming requires further research, taking anthropometric indicators of the lower limbs into account.

Our research indicates that the amplitude of toes movements was higher in boys. As mentioned, in both sexes, the frequency of underwater undulatory swimming was similar. Atkison and Nolte [3] equate the product of these two variables ( $A$ and $f$ ) with the vertical velocity of the toes. They claim that its value largely determines underwater swimming velocity. It is known that the ability to obtain high vertical velocity of the toes is the basis for effective movement using the underwater undulatory swimming technique [4], [10]. In this study, the values of the product de- fined in this way were: 1.05 (girls) and 1.15 (boys). As it can be seen, our results are based on the observations described above. Additional confirmation is provided by correlation analysis, in which the relationship between $I_{\mathrm{Af}}$ and $v_{\mathrm{COM}}$ was positive for both sexes, although it should be noted that in the group of girls, this relationship was not statistically significant.

In this study, girls reported slightly larger maximal flexion and extension of the ankle joints than boys, which affected the significant differences in the range of motion of the ankle joints. This is probably connected with the dimorphic differences in the level of flexibility. As reported, among others, by Alter [1], women have lower muscle mass, they are characterised by different joint geometry and collagen structure, which results in a greater range of mobility in the joints compared to men. Many authors believe that without adequate flexibility, it is impossible to achieve high swimming results [1]. There are publications indicating that in the case of underwater undulatory swimming, limiting movement in the ankle joints has negative consequences in the form of increasing flexion in the knee joints, resulting in a decrease of velocity [22]. Our results are in contrast to these data. It was found that with significant $A_{\text {ROM }}$ differences, the range of motion in the knee joints of boys and girls performing underwater undulatory swimming was similar. It cannot be ruled out that lower $A_{\text {ROM }}$ values in the boys' group were associated with an increase in extensional movement in hip joints. This possibility was suggested, among others, by Higgs et al. [10]. Unfortunately, this issue was not the purpose of research. It was focused on indicators characterising the movement of the whole body (e.g., velocity, distance per cycle and frequency) and detailed feet movements (amplitude, values of angles in the ankle joints, horizontal displacement of toes during downbeat). Mobility of the knee joints was also taken into account. The applied method was developed on the basis of the literature, which showed that the movement of the feet and angular changes in the ankle joints most significantly determine the effectiveness of underwater undulatory swimming [2], [4], [10]. In the course of analyses, it turned out that the adopted methodology had limitations. Hence, in subsequent studies in this area, the course of movement in the hip joints should also be taken into account.

The aim of this study was also to characterise the relationships between selected kinematic variables and the velocity of underwater undulatory swimming, including division into sex. In the case of this motor task, it should be born in mind that the competitor 
performs movements in many joints at the same time. There are theories known, according to which mechanical energy is transmitted through the subsequent segments of the body from the trunk towards the feet, which allows for an increase in the efficiency of underwater undulatory swimming [20]. This means that the course of movement in knee or ankle joints may be dependent on the quality of movement, e.g., in the hip joints. Therefore, the analysis of a single element of the technique of underwater undulatory swimming should be performed while also considering segments which seemingly do not concern the analysis.

In the present study, it turned out that the directions of dependence between $v_{\mathrm{COM}}$ and kinematic variables were similar in both sexes. The only significant difference was noted in the case of amplitude $(A)$. Among girls, this relationship was negative $(r=$ $-0.137)$, while in boys it was positive $(r=0.291)$. Both results, however, were not statistically significant, which coincides with earlier reports stating that the amplitude of the toes does not determine the velocity of underwater undulatory swimming [2], [3].

In both sexes, the directions of dependence of other variables with $v_{\mathrm{COM}}$ were the same. The values of the majority of correlation coefficients were comparable, although a few significant disproportions were observed. In the female group, the range of motion in the knee joints was an indicator strongly negatively correlated with the velocity of underwater undulatory swimming $(r=-0,714)$. In turn, in boys, the relationship between $K_{\mathrm{ROM}}$ and $v_{\mathrm{COM}}$ was weak $(r=$ $-0.281)$. It has already been documented that excessive flexion in the knee joints has a negative effect on the velocity of underwater undulatory swimming [2], [21]. According to Higgs et al. [10], higher efficiency of underwater undulatory swimming can be obtained by increasing the range of motion in the hip joints during the upward movement of the feet. Perhaps, the examined boys presented a greater range of motion in the aforementioned joints, which would also explain the previously discussed higher amplitude of the foot movements recorded in this group.

In this study, the relationship between maximal flexion of the ankle joints $\left(A_{\mathrm{FLEX}}\right)$ and the horizontal velocity of swimmers was negative, although the strength of these correlations was different for girls and boys ( $r=-0,501$ and $r=-0.206$, respectively). The fact that maximising the extension of the ankle joints at the expense of flexion of the ankle joints is an effective strategy in increasing the velocity when performing underwater undulatory swimming was indicated by Wądrzyk et al. [21]. This study also showed that the range of motion in the ankle joints did not significantly affect $v_{\mathrm{COM}}$. The mentioned observation is a supplement to the characteristics of the movement relationships in the ankle joint with the velocity obtained during underwater undulatory swimming.

A variable particularly associated with $v_{\mathrm{COM}}$ was the horizontal displacement of toes during downbeat - Toe $_{\text {DISP }}(r=-0.817$ in girls and $r=-0.610$ in boys). According to Arellano et al. [2], competitors who achieve lower underwater swimming velocity while moving their foot downwards, clearly also move it backwards. This is due to the incorrect positioning of its main propelling surface, thus, the efficiency of extending the knee joints is low. It seems that the foot movement path may also be dependent on the socalled "feeling of the water" [19]. Although the term has been commonly used by coaches almost from the beginning of the 20th century, in scientific research on swimming, it is often not included [18]. It is identified with the processes of proper reception of stimuli from the aquatic environment and an adequate response to them, therefore, in biomechanical measurements, this problem is usually neglected [18]. The kinematic indicator used to assess the sensation of water in swimming full strokes can be the horizontal displacement of the hand backwards during underwater movement of the upper limb. At best, the hand should not move relative to water in this direction [8]. In this situation, it is believed that the swimmers' movements are highly effective, impossible to achieve without sufficiently high feeling of the water [8], [26]. It seems that an adequate indicator for assessing the sensation of water of the lower limbs in underwater undulatory swimming can be measured in the present study by horizontal displacement of toes during downbeat. Of course, there is no possibility of total similarity to the full stroke swimming because the movement capabilities of individual segments of the limbs (upper and lower) and the anatomical structure of their distal segments (foot and hand) differ significantly [1]. It is probable that due to the mentioned difficulties, the "feeling of water" in relation to the distal segment of the lower limb is still not described in detail.

In the case of underwater undulatory swimming, it has been reported that it is possible to propel the body forward without moving the toes backwards in relation to the water [4]. Ungerechts and Arellano [20] dealt with the explanation of this issue, according to which this phenomenon can be attributed to the socalled vortex propulsion. In this study, in both groups, during the downward movement of the feet, their toes moved backwards. Differences in the Toe DISP $_{\text {index }}$ among girls and boys were small (about $0.02 \mathrm{~m}$ ), thus presumably, the value of horizontal displacement of 
toes during downbeat was not affected by the genderspecific physiological or anthropometric disparities that were discussed earlier. At the same time, in both groups, there was a strong negative correlation between Toe DISP $_{\text {and }} v_{\text {COM }}$. Therefore, it seems that the feeling of water is also important when propelling the body with only the lower limbs.

The velocity of underwater undulatory swimming was also dependent on another indicator - distance per cycle (DPC). In the case of swimmers of both sexes, higher DPC value resulted in higher horizontal velocity of the center of mass. Other authors also came to similar conclusions [2]. However, changes in the frequency of movements should be monitored with the DPC measurement. It may turn out that maximising the distance per cycle may considerably extend the cycle time [21]. This, in turn, being synonymous with a decrease in the frequency of movements, may negatively affect the velocity of the swimmer [7].

Analysis of the results allows to conclude that in both groups, the relationships between kinematic variables and the velocity obtained during underwater undulatory swimming were very similar. The observed differences between genders in $v_{\mathrm{COM}}$ determinants were small, therefore, the assessment and possible correction of technique of the discussed movement mode in both groups should emphasise the same elements.

\section{Conclusions}

This study provides information enabling verification of the erected hypotheses. On their basis, it can be stated, among others, that boys swim faster with the use of underwater undulatory swimming. In addition, both sexes differ in the level of kinematic indicators describing the technique of underwater swimming. The movements performed by girls are characterised by a lower amplitude of the toes, shorter distance per cycle and lower values of the amplitude and frequency product. In addition, female swimmers present greater range of motion in the ankle joints. The results of this study also provide information that in both sexes, the effectiveness of underwater undulatory swimming can be described using the same kinematic variables. The indicators influencing a swimmer's velocity include maximal extension of the ankle joints, distance per cycle and horizontal displacement of toes during downbeat. This means that the technical training of underwater undulatory swimming can be implemented without dividing it into gender. At the same time, the further direction of research should be to determine the impact of strength and power of the lower limbs and somatic structure on the effectiveness of underwater undulatory swimming.

\section{Funding details}

This work was supported by the University of Physical Education in Kraków under the Grant number 110/MN/INB/2018.

\section{Acknowledgements}

We would like to kindly thank the Sports Championship School in Oświęcim and all of the swimmers taking part in the study. In particular, the authors of the work would like to thank Mr. Marek Dorywalski for help in organising the research.

\section{Disclosure statement}

No potential conflict of interest was reported by the authors.

\section{References}

[1] Alter M.J., Science of flexibility, Human Kinetics, 2004.

[2] Arellano R., Pardillo S., Gavilan A., Underwater undulatory swimming: kinematic characteristics, vortex generation and application during the start, turn and swimming strokes, [in:] K.E. Gianikellis (Ed.), Proceedings of the XXth International Symposium on Biomechanics in Sports, University of Extremadura, 2002.

[3] AtKison R.R., Nolte V., Kinematic comparison of the underwater dolphin kick between swimmers with different levels of competitive ability, [in:] The Proceedings of the 16th Biannual Conference of The Canadian Society for Biomechanics, Queen's University, 2010, 149.

[4] AtKison R.R., Dickey J.P., Dragunas A., Nolte V., Importance of sagittal kick symmetry for underwater dolphin kick performance, Hum. Mov. Sci., 2014, 33, 298-311, DOI: 10.1016/j.humov.2013.08.013.

[5] Bernardina G.R., Cerveri P., Barros R.M., Marins J.C., Silvatti A.P., Action sport cameras as an instrument to perform a 3D underwater motion analysis, PLoS One, 2016, 11(8), 1-14, DOI: 10.1371/journal.pone.0160490.

[6] Beunen G., Malina R., Growth and biologic maturation: relevance to athletic performance, [in:] $\mathrm{H}$. Hebestreit, O. Bar-Or (Eds.), The young athlete: 3-17, Blackwell Publishing, Ltd., 2008.

[7] Cohen R.C., Cleary P.W., Mason B.R., Simulations of dolphin kick swimming using smoothed particle hydrodynamics, Hum. Mov. Sci., 2012, 31(3), 604-619, DOI: 10.1016/ j.humov.2011.06.008.

[8] Gorgoulis V., Antoniou P., Aggeloussis N., Mavridis G., Kasimatis P., Vezos N., Boli A., Mavromatis G., Kinematic characteristics of the stroke and orientation of the hand during front crawl resisted swimming, J. Sports Sci., 2010, 11, 1165-1173, DOI: 10.1080/02640414.2010.50725. 
[9] Higgs A.J., Pease D.L., Sanders R., Kinematic differences between upkick and downkick in undulatory underwater swimming, [in:] F. Colloud, M. Domalain, T. Monnet (Eds.), Proceedings of the 33rd Conference of the International Society of Biomechanics in Sports, International Society of Biomechanics in Sports, 2015, 960-963.

[10] Higgs A.J., Pease D.L., Sanders R., Relationships between kinematics and undulatory underwater swimming performance, J. Sports Sci., 2017, 35(10), 995-1003, DOI: 10.1080/ 02640414.2016.1208836.

[11] Kenney W.L., Wilmore J.H., Costill D.L., Physiology of sport and exercise, sixth ed., Human Kinetics, 2015.

[12] KJendlie P.L., Stallman R., Morphology and swimming performance, [in:] L. Seifert, D. Chollet, I. Mujika (Eds.), World book of swimming: from science to performance, Nova Science Publishers Inc, 2011, 203-221.

[13] Mooney R., Corley G., Godfrey A., Osborough C., Quinlan L., Òlaighin G., Application of video-based methods for competitive swimming analysis: a systematic review, Sport Exerc. Med. Open J., 2015, 1(5), 133-150, DOI: 10.17140/SEMOJ-1-121.

[14] Nevill A.M., Whyte G.P., Holder L.R., Peyrebrune M., Are there limits to swimming world records?, Int. J. Sports Med., 2007, 28(12), 1012-1017.

[15] Nevill A.M., OxFord S., DunCAn M.J., Optimal body size and limb-length ratios associated with 100-m PB swim speeds, Med. Sci. Sports Exerc., 2015, 47 (8), 1714-1718, DOI: 10.1249/ MSS.0000000000000586.

[16] Pelayo P., Alberty M., The history of swimming research, [in:] L. Seifert, D. Chollet, I. Mujika (Eds.), World book of swimming: from science to performance: $X I X-X V I$, Nova Science Publishers, Inc, 2011.

[17] Seifert L., Boulesteix L., Chollet D., Effect of gender on the adaptation of arm coordination in front crawl, Int. J. Sports Med., 2004, 25 (3), 217-223.

[18] Starosta W., Term of "water feeling”, its significance, structure, changeability, formation and conditions in opinion of athletes of different water sports and its coaches, [in:] W. Starosta
(Ed.), Kinestesis - new method of development highest quality of movements, International Association of Sports Kinetics, 2015, 229-268 (in Polish).

[19] StrZaŁa M., KrężaŁeK P., KaCa M., GŁąB G., Ostrowski A., Stanula A., TyKa A., Swimming speed of the breaststroke kick, J. Hum. Kinet., 2012, 35, 133-139, DOI: 10.2478/ v10078-012-0087-4.

[20] Ungerechts B., Arellano R., Hydrodynamics in swimming, [in:] L. Seifert, D. Chollet, I. Mujika (Eds.), World book of swimming: from science to performance, Nova Science Publishers, Inc, 2011, 21-41,

[21] Wądrzyk Ł., Nosiadek L., Staszkiewicz R., Underwater dolphin kicks of young swimmers - evaluation of effectiveness based on kinematic analysis, Hum. Mov., 2017, 18(4), 23-29, DOI: 10.1515/humo-2017-0030.

[22] Willems T.M., Cornelis J.A. De Deurwaerder L.E., RoElandT F., DE Mits S., The effect of ankle muscle strength and flexibility on dolphin kick performance in competitive swimmers, Hum. Mov. Sci., 2015, 36, 167-176, DOI: 10.1016/j.humov.2014.05.004.

[23] WolańSKi N., Human biological development. Basics of auxology, gerontology and health promotion, Eighth Ed., Wydawnictwo Naukowe PWN, Warszawa 2012 (in Polish).

[24] Wolfrum M., Rust C.A., Rosemann T., Lepers R., KNECHTLE B., Changes in breaststroke swimming performances in national and international athletes competing between 1994 and 2011 - a comparison with freestyle swimming performances, BMC Sports Sci. Med. Rehabil., 2014, 6 (18), DOI: 10.1186/2052-1847-6-18.

[25] von Loebbecke A., Mittal R., Fish F., Mark R., A comparison of the kinematics of the dolphin kick in humans and cetaceans, Hum. Mov. Sci., 2009, 28 (1), 99-112, DOI: 10.1016/j.humov.2008.07.005.

[26] Zatoń K., Szczepan S., Kazimierów R., Rejman M., Advisability on the shift from standard front crawl swimming technique to the "kayaking" and "loping" variants, Hum. Mov., 2012, 13 (3), 225-235, DOI: 10.2478/v10038-012-0026-1. 